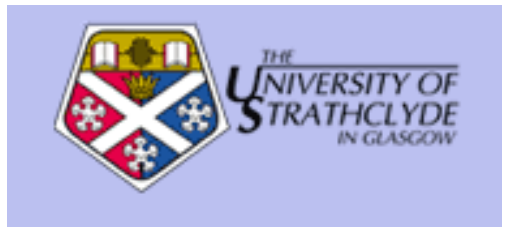

Muscat, M. and Degiorgio, K. and Wood, J. (2009) Comparison between different approaches for the evaluation of the hot spot structural stress in welded pressure vessel components. In: ASME Pressure Vessels and Piping Conference, 26-30 July 2009, Prague, Czech Republic.

http://strathprints.strath.ac.uk/20223/

Strathprints is designed to allow users to access the research output of the University of Strathclyde. Copyright (C) and Moral Rights for the papers on this site are retained by the individual authors and/or other copyright owners. You may not engage in further distribution of the material for any profitmaking activities or any commercial gain. You may freely distribute both the url (http://strathprints.strath.ac.uk) and the content of this paper for research or study, educational, or not-for-profit purposes without prior permission or charge. You may freely distribute the url (http://strathprints.strath.ac.uk) of the Strathprints website.

Any correspondence concerning this service should be sent to The Strathprints Administrator: eprints@cis.strath.ac.uk 


\section{COMPARISON BETWEEN DIFFERENT APPROACHES FOR THE EVALUATION OF THE HOT SPOT STRUCTURAL STRESS IN WELDED PRESSURE VESSEL COMPONENTS}

\author{
Martin Muscat \\ University of Malta \\ Faculty of Engineering \\ Mechanical Engineering \\ Msida, Tal-Qroqq \\ Malta \\ Phone: +356 23402392 \\ E-mail:mmmusc@eng.um.edu.mt
}

\author{
Kevin Degiorgio \\ University of Malta \\ Faculty of Engineering \\ Mechanical Engineering \\ Msida, Tal-Qroqq \\ Malta \\ Phone: +356 23402392 \\ E-mail:kdeg0002@ um.edu.mt
}

\author{
James Wood \\ University of Strathclyde \\ Mechancial Engineering \\ James Weir Building \\ 75 Montrose Street \\ Glasgow, United Kingdom \\ Phone: +44 (0)141 5482043 \\ E-mail:j.wood@strath.ac.uk
}

\begin{abstract}
Fatigue cracks in welds often occur at the toe of a weld where stresses are difficult to calculate at the design stage. To circumvent this problem the ASME Boiler and PV code Section VIII Division 2 Part 5 [1] uses the structural stress normal to the expected crack to predict fatigue life using elastic analysis and as welded fatigue curves. The European Unfired Pressure Vessel Code [2] uses a similar approach. The structural stress excludes the notch stress at the weld toe itself. The predicted fatigue life has a strong dependency on the calculated value of structural stress. This emphasizes the importance of having a unique and robust way of extracting the structural stress from elastic finite element results. Different methods are available for the computation of the structural hotspot stress at welded joints. These are based on the extrapolation of surface stresses close to the weld toe, on the linearisation of stresses in the through-thickness direction or on the equilibrium of nodal forces. This paper takes a critical view on the various methods and investigates the effects of the mesh quality on the value of the structural stress. T-shaped welded plates under bending are considered as a means for illustration.
\end{abstract}

\section{NOMENCLATURE}

$\sigma_{\max }$ Maximum stress within one complete cycle.

$\sigma_{\min }$ Minimum stress within one complete cycle.

$\Delta \sigma \quad$ is given by $\sigma_{\max }-\sigma_{\min }$ $\sigma_{\text {nom }} \quad$ Nominal stress. Calculated using strength of materials fomulae

$\sigma_{s} \quad$ Structural stress. Is given by $\sigma_{m}+\sigma_{b}$

$\sigma_{m} \quad$ Membrane stress, is the component of the structural stress that is uniformly distributed and equal to the average value of stress across the section thickness.

$\sigma_{b} \quad$ Bending stress, is the component of the structural stress that varies linearly across the section thickness.

$\sigma_{t} \quad$ Total stress

$\sigma_{i j}^{m} \quad$ is the membrane stress component (for stress classification), three direct and three shear

$\sigma_{i j}$ is the stress component along a classification line

$x \quad$ is the coordinate along a classification line

$h \quad$ is the wall thickness at the linearisation line/plane

$\sigma_{i j 1,2}^{b}$ are the bending stress components at each end of the classification line. $\left(\sigma_{i j 2}^{b}=-\sigma_{i j 2}^{b}\right)$

$\sigma_{i j 1,2}^{p}$ are the peak stress components at each end of the classification line.

$\sigma_{i j 1,2}$ are the total stress components at each end of the classification line. 
$\sigma_{h s} \quad$ is the extrapolated structural stress at a hot spot

$\sigma_{0.4 t}$ is a stress component at a distance of $0.4 t$ away from the hot spot. Similar definition for $\sigma_{0.9 t}, \sigma_{1.4 t}$

$t \quad$ is the wall thickness of a welded plate. Used for stress extrapolation eauations.

$\delta \quad$ is the distance between reference planes A-A and B-B used in Dong's nodal force method.

$f_{x^{\prime}}, f_{y^{\prime}}, f_{z^{\prime}}$ The sectional forces in the local coordinate system used in the nodal force approach.

$m_{y^{\prime}} \quad$ The sectional moment in the local coordinate system used in the nodal force approach.

$S_{\text {int }} \quad$ Stress intensity

$x t r \_q \quad$ Extrapolated stresses, quadrilateral shaped elements

$x t r \_t \quad$ Extrapolated stresses, triangular shaped elements

lin_q Linearized stresses, quadrilateral shaped elements

lin $t \quad$ Linearized stresses, triangular shaped elements

$n o \bar{d} \_q$ Nodal force method stresses, quadrilateral shaped elements

nod_t Nodal force method stresses, triangular shaped elements

\section{INTRODUCTION}

The stress range is the main parameter that needs to be determined when performing a fatigue analysis. Accuracy in its value is fundamental to achieving representative fatigue lives. The stress range $\Delta \sigma$ is the difference between the maximum and minimum stress values within one complete cycle. The stress range is historically determined in terms of either one of the following - nominal stress, notch stress and structural stress. The type of stress used to obtain the cyclic stress range must be consistent with the fatigue data represented in the applied fatigue curves and what the particular Code requires for the detail under consideration.

The nominal stress $\sigma_{\text {nom }}$ approach has the drawback in that it largely ignores the actual dimensional variations of a particular structural detail. An alternative is to use the structural stress, $\sigma_{s}$, approach. The structural stress includes the stress concentrating effects of the detail itself but not the local nonlinear stress peak caused by the notch at the weld. The notch effect is included in the hot spot S-N curve determined experimentally [3] and this is why it must be removed from the FEA results. The Code allowables are defined in terms of the applied experimental field stresses. A hot spot refers to a critical point in the component where fatigue cracking is most likely to occur. A hot spot structural stress refers to the value of structural stress determined at a hot spot. The main effect of a notch is to produce a non-linearity in the stress distribution. The total stress combines the structural stress together with the effects of a stress raiser. The additional stress to the structural part that forms the notch stress is referred to as the non- linearity stress as shown in Fig.1. Over the years researchers have looked into finding methods to calculate structural stresses. Some of the developed methods include - Stress linearization through thickness, surface stress extrapolation and the nodal force (Dong's) approach [13]. It can be said that the structural stress is a somewhat 'fictitious' stress, it cannot be taken directly from the stresses at a point and can be obtained only through calculations.

\section{METHODS TO CALCULATE THE STRUCTURAL STRESS}

\section{Stress linearization}

Stress categorization in Design by Analysis breaks down the total elastic stress into membrane, bending and peak stresses. When the analysis is based on thin shell finite elements, then there is no difficulty in identifying membrane and bending stresses, as these are part of the underlying theory. The membrane plus bending stress obtained through stress linearization results in the structural stress.

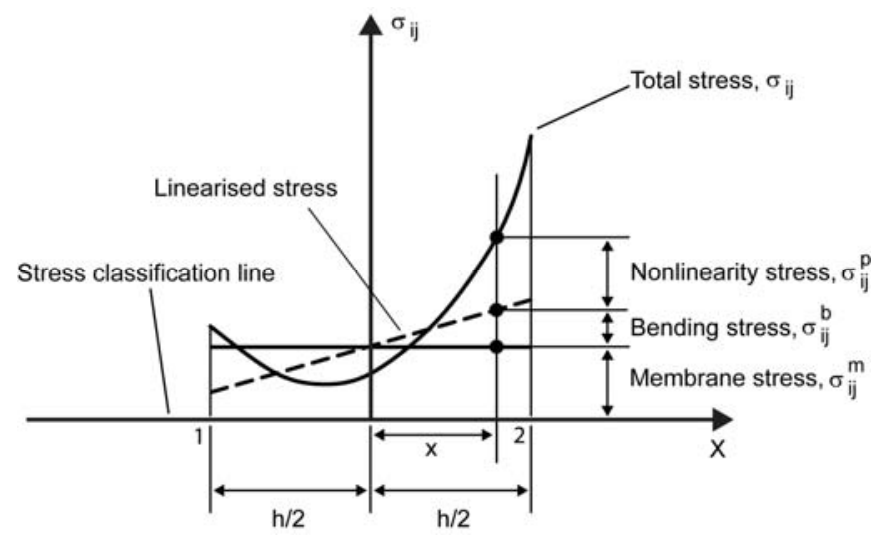

Figure 1 - Typical stress distribution along a classification line

Stress linearization and categorisation is performed by first selecting a classification line along which the linearization is carried out. A procedure for carrying out the stress linearization was first proposed by Kroenke [4] [5] and is still in use to this day with some modifications. Figure 1 shows a typical stress distribution along a classification line. The linearization procedure is computed at the stress components level $\sigma_{i j}$. When analysing axisymmetric cases, in Kroenke's procedure the shell wall is assumed to be straight. However this is not always the case. To cater for these cases Gordon [6] suggested a modification to Kroenke's procedure. In principle the procedure is the same with the difference that the position of the neutral axis is shifted [7]. When performing stress linearization using finite element tools, one must be aware of stress averaging. To compute the stress at the node representing the hot spot, for example at a weld toe, post- 
processors normally compute an average value between the lower stresses in the weld material and the higher stresses immediately in front. Thus the computed stresses at the hot spot can be under-estimated. To avoid this problem the linearization procedure should be based on those elements directly in front of the hot spot [8].

\section{Surface stress extrapolation}

Surface stress extrapolation is used to obtain the structural stress at the hot spot. Fatigue design rules based on this approach were published by various entities [9]. The objective is to remove the non-linear peak stress as shown in Fig.2. In concept, this approach looks at the stress distribution approaching the hot spot and extrapolates these stresses to it [3]. The stress distribution in the vicinity of the hot spot can be obtained using finite element analysis with a linear elastic material model or with using strain gauges.

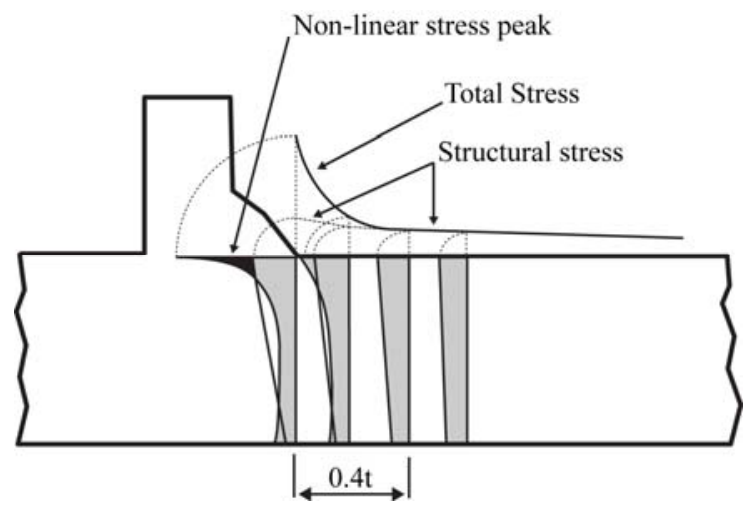

Figure 2 - Surface distribution across the plate thickness and along the surface in the vicinity of the weld, adapted from [3]

The International Institute of Welding (IIW) has published detailed guidelines on the extrapolation procedures [3, 10]. These IIW guidelines introduce the notion of type ' $a$ ' and type ' $b$ ' hot-spots, with different procedures in their assessment. Type ' $a$ ' hot spots occur on a plate surface while type ' $b$ ' hot spots occur on a plate edge. It has been shown $[3,11,12]$ that detailed rules for finite element modelling and stress evaluation are necessary to avoid large scatter and uncertainties in the computed structural stresses when using surface stress extrapolation.

The extrapolation can be either linear or quadratic. The ideal method for stress extrapolation would be to first extrapolate the stress components and then calculate the principal stresses or stress intensities accordingly. Different extrapolation equations are given in the literature for extrapolating the stress components [3]. For example for type 'a' hot spots and for a relatively fine mesh with stresses taken at nodal points, the hot spot stress $\sigma_{h s}$ is given by

$$
\sigma_{h s}=2.52 \sigma_{0.4 t}-2.24 \sigma_{0.9 t}+0.72 \sigma_{1.4 t}
$$

When creating a finite element model it is often the case that the elements size does not allow the extrapolation points to be in the exact position as required by extrapolation equations. In such cases curve fitting through the available points is performed to obtain the stresses at the required position. Guidelines also make a distinction between fine and coarse meshes and in some cases specify the use of midside nodes. The guidance is also different for shells and welds. In addition there is no requirement that the FEA results should be converged in the vicinity of the singularity. Indeed it may well be the case that such convergence would invalidate the approach.

\section{Nodal force (Dong's) Approach}

Dong [13] proposed an alternative method for calculating the structural stress at the hot spot. The method is based on nodal force equilibrium. In the case of solid models, the method makes use of through thickness stress distribution at some distance $\delta$ from the hot spot. From equilibrium considerations, the additional bending stresses that are produced by through thickness shear stresses at the hot spot are then added to the through thickness stress to obtain the hot spot structural stress $[8,12]$. The procedure is considered to be rather mesh insensitive since stresses are taken from a distance $\delta$, away from the hot spot.

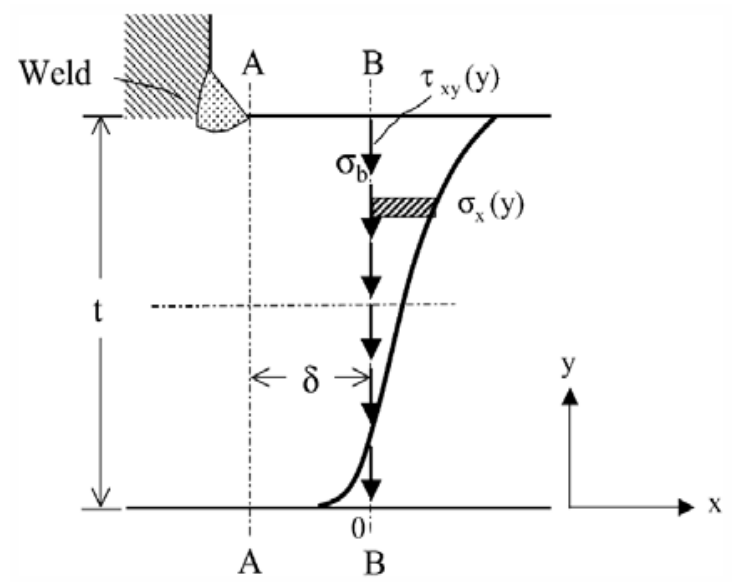

Figure 3 - Structural stress calculation for through thickness fatigue crack, reproduced from Dong [13]

In Fig. 3 considering equilibrium conditions between section $\mathrm{A}-\mathrm{A}$ and section $\mathrm{B}-\mathrm{B}$, the membrane and bending components of the structural stress at section AA, $\sigma_{m}$ and $\sigma_{b}$, can be calculated from equations (2) and (3).

$$
\sigma_{m}=\frac{1}{t} \int_{0}^{t} \sigma_{x}(y) d y
$$




$$
\sigma_{m} \frac{t^{2}}{2}+\sigma_{b} \frac{t^{2}}{6}=\int_{0}^{t} \sigma_{x}(y) y d y+\delta \int_{0}^{t} \tau_{x y}(y) d y
$$

Equation (2) represents the force balances in the $\mathrm{x}$-direction, evaluated along B-B and equation (3) represents moment balances with respect to A-A at $\mathrm{y}=0 . \sigma_{x}(y)$ is the stress in the $\mathrm{x}$-direction along section $\mathrm{B}-\mathrm{B}$.

Equations (2) and (3) are applicable to cases of solid models with monotonic through-thickness stress distribution [11]. When the stress distribution through thickness is nonmonotonic and is symmetric a linearization depth of $t / 2$ is proposed $[11,13]$. Such an example occurs for the case of twosided geometrically symmetrical welds under symmetrical loading. In this approach a different formulation to the one presented in equations 2 and 3 is used [13].

Furthermore in certain cases, linearization up to a depth of $t_{1}<t$ is recommended. Such a case occurs for welds at plate edges with $t_{1}$ representing the final crack length taken as a fatigue failure criterion.

For the case of shell models Dong [13] presents two general methods for structural stress calculations. One method uses stresses and stress resultants while the other uses element nodal forces. For three dimensional shell elements Dong [13] presents the structural stress at the weld toe as in equation (4).

$$
\sigma_{s}=\sigma_{m}+\sigma_{b}=\frac{f_{x^{\prime}}}{t}+\frac{6\left(m_{y^{\prime}}+\delta f_{z^{\prime}}\right)}{t^{2}}
$$

Equation (4) is similar to equations (2) and (3) and uses the approach of stresses and stress resultants. The method uses nodal forces in the local coordinate system of the shell elements at section A-A (Fig. 3) and calculates the corresponding sectional forces and moments $\left(f_{x}^{\prime}, f_{y}^{\prime}, m_{y^{\prime}}\right)$ so that the structural stress at the weld toe is then given by equation (5), [13].

$$
\sigma_{s}=\sigma_{m}+\sigma_{b}=\frac{f_{x^{\prime}}}{t}+\frac{6 m_{y^{\prime}}}{t^{2}}
$$

For solid elements the sectional or distributed forces and moments can be computed along the weld toe line from the nodal forces and moments [13]. This can be done using appropriate shape functions and using the idea of work equivalence. [10, 12].

\section{COMPARATIVE STUDY ON METHODS CALCULATE THE STRUCTURAL STRESS}

In this work the objective was to investigate the effects of various finite element model variations on the calculated structural stresses. These variations included different geometric and loading combinations together with the effects of mesh size and element type. Several similar exercises have been carried out by a number of authors. Some of the works include those by Poutiainen [8], Fricke [11] and Doerk [12].

\section{Stress linearisation}

Stress linearization was carried out using the linearization tool included in finite element software ANSYS [14]. The technique is similar to that reported by Gordon [6]. Following a well established guideline [8] the elements in front of the hot spot were selected. This avoids the risk of having under estimated results due to stress averaging. Stress components are linearised and categorised so that the membrane plus bending stress intensity $\left(S_{i n t}\right)$ is calculated at the hot spot location.

\section{Surface stress extrapolation}

The surface stress extrapolation was carried out in quadratic form. The surface extrapolation points were taken at distances of $0.4 t, 0.9 t$ and $1.4 t$ away from the hot spot respectively. The mesh size was pre-arranged at the modelling stage so that there were nodes at the $0.4 t, 0.9 t$ and $1.4 t$ positions. The procedure adopted in this work was to extract the stresses from the nodal results. Because of this, the extracted stresses are averaged results. Each stress component is individually extrapolated to obtain the structural stress value of all the stress components at the hot spot. Extrapolation of the stresses was done by fitting a quadratic curve to the stress component values at the three extrapolation points. The structural stress intensity $\left(S_{\text {int }}\right)$ is thus calculated from the structural stress components.

\section{Nodal force method}

The ASME Boiler and pressure vessel code 2007 Section VIII Division 2 Part 5 gives equations to compute the membrane and bending stresses from element nodal internal forces both for solid continuum elements and for shell elements. This is the recommended method when internal nodal forces can be obtained as part of the finite element output since the results are insensitive to mesh density [1].

\section{Finite element model}

In order to compare the structural stress computed using the linearization through thickness, surface extrapolation and the nodal force (Dong's) method, T-shaped welded plates under bending are considered in this paper. The geometry including the boundary conditions and applied loading is shown in Fig. 4. The hot spot of interest is the weld toe. The model used 8noded quadratic elements having different mesh sizes and element shapes. The weld itself was meshed in two different ways once using quadrilaterally shaped elements and then using triangularly shaped elements.

Table 1 describes the number of elements used through thickness (e_t) and along the surface $\left(e_{-} s_{i}, i=1,2,3\right)$.

Tables A.1 and A.2 in Annex A show the resulting element meshes. In the finite element meshes there are two variables, the number of elements in the thickness direction, $e_{-} t$ and the number of elements in the $0-0.4 t, 0.4 t-0.9 t$, and $0.9 t-1.4 t$ 
regions along the surface, e_s, referred respectively as e_ $\mathrm{s}_{1}$, e_ $\mathrm{s}_{2}$, and $\mathrm{e}_{-} \mathrm{s}_{3}$.

From the figures shown in Tables A.1 and A.2 it can be immediately observed that some of the meshes are not acceptable. For example in some cases the mesh is too coarse while the element aspect ratio and element transition sizes are poor. The mixture of good and poor meshes was deliberate. It may be argued that when performing a finite element analysis, it is essential that before interpreting the stress results it should be ascertained that the resulting solution is a converged solution. This can be made by refining the mesh and comparing the resulting stresses or else by using mesh quality measures to ascertain whether results locally are acceptable. The objective of the study is not to deviate from the importance of having a converged solution but rather to study the mesh sensitivity of the methods available for calculating the structural stress. When performing an elastic finite element analysis at a notch singularity it is observed that mesh refinement results in a higher value of the total stress. This is implies that the value of total stress at the hot spot will affect the stress distribution in the elements around the hot spot. Clearly the structural stress method used, can have an affect on the calculated structural stress.

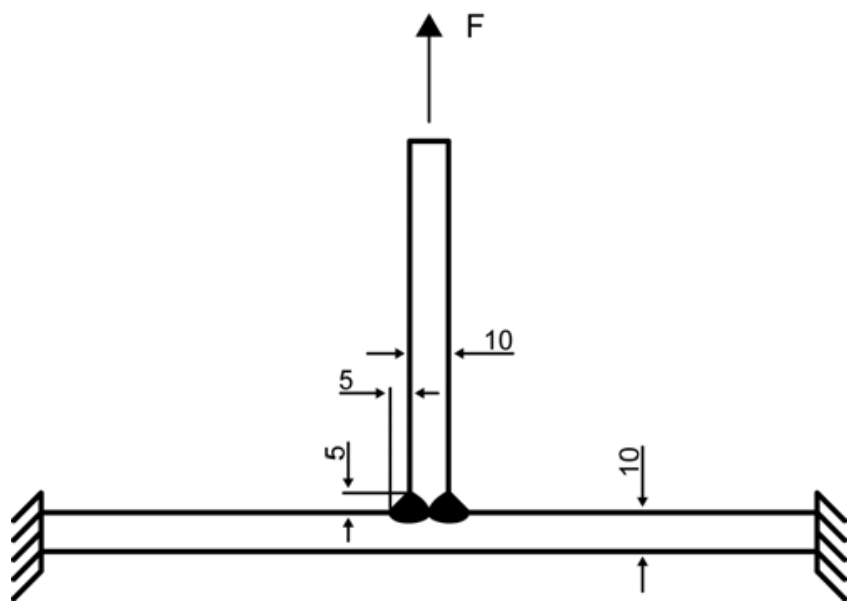

Figure 4 - T shaped welded plates under bending

\section{Analysis steps}

A finite element solution was carried out for all combinations of element meshes. For each solution the structural stress was calculated using the linearization through thickness method, the surface stress extrapolation method and the method based on nodal forces as presented in the ASME B\&PVC [1]. For stress linearization only the elements in front of the hot spot were used. This avoids the risk of having under-estimated results caused by averaging of stresses due to adjacent elements. To see the effect that this averaging procedure has on the results, the analyses were repeated with the linearization carried out using stress averaging of the adjacent elements across the classification line.

\section{Results}

Fig. A.1 illustrates graphically the calculated structural stresses for the different meshes used. Though the graphs are line type graphs, the intention of the lines is not to relate the various results together but rather to simplify the interpretation of the results by highlighting the trend the results are taking for different meshes.

Table 1 - Number of elements through thickness and along surface at the hot spot

\begin{tabular}{|c|c|c|c|c|}
\hline \multirow{3}{*}{$\begin{array}{c}\text { Elements } \\
\text { through } \\
\text { thickness, e_t }\end{array}$} & \multicolumn{4}{|c|}{ Elements along surface, e_s } \\
\hline & e_s & e_s $s_{1}$ & e_s $\mathbf{s}_{2}$ & e_s $\mathbf{S}_{3}$ \\
\hline & & $0-0.4 t$ & $0.4 t-0.9 t$ & $0.9 t-1.4 t$ \\
\hline \multirow{5}{*}{$2,4,8,10$} & A & 1 & 1 & 1 \\
\hline & B & 1 & 2 & 2 \\
\hline & $\mathrm{C}$ & 2 & 2 & 2 \\
\hline & $\mathrm{D}$ & 2 & 3 & 3 \\
\hline & $\mathrm{E}$ & 3 & 3 & 3 \\
\hline
\end{tabular}

\section{Discussion of Results}

With regards to surface stress extrapolation the structural stresses computed using different mesh designs fluctuate considerably but appear to be more stable for a model having 4 or more elements through thickness for cases $\mathrm{C}, \mathrm{D}$ and $\mathrm{E}$ described in Table 1.

When using unaveraged stresses and linearization through thickness, the structural stresses computed using different mesh designs are stable for models having 4 or more elements through thickness for all cases A, B, C, D, E described in Table 1. This is not so when averaged stresses are used and the elements modeling the weld are triangular in shape (Fig.A.2).

Comparing structural stresses calculated using linearisation through thickness with those obtained using surface extrapolation and the nodal force method it is observed that the former calculated stresses are always slightly higher by about $4 \%$.

\section{The nodal force (Dong's) method}

Structural stresses calculated using the nodal force method show that the method is mesh insensitive for the models of the T-shaped welded plates under bending considered in the paper.

\section{Linearization through thickness}

\section{Effect of the element shape}

The structural stress obtained from the linearization through thickness procedure was generally unaffected by the element shape (quadrilateral or triangular) used to model the weld, Fig. A.1. The elements used were 8-noded solid elements (Plane82), having mid-side nodes along the sides. Plane82 
elements give accurate results for mixed meshes (quadrilateral and triangular) and can tolerate irregular shapes without much loss of accuracy [14]. The largest difference occurs in models in which the number of elements in the thickness direction is two, e $\mathrm{t}=2$. It is likely that the cause for this result is a combination of the geometry, element type and mesh density of the modelled weld that then reflects in different stress distributions in the thickness direction. At $e_{-} t=4$ the difference in the linearized stress values becomes very small (less than $2 \%$ ) and disappears altogether at $\mathrm{e}_{-} \mathrm{t}=8$ and 10 . Therefore mesh refinement through thickness appears to close the gap between the two element shapes used to represent the weld.

\section{Element size and distortion}

Though clearly a number of models produced poor meshes at the weld, the effect on the structural stress values is primarily observed when $e_{-} t=2$, i.e. when there are two elements in the thickness direction. This reflects in higher calculated structural stresses. The difference is about $4 \%$ (Fig.A.1). When the number of elements in the thickness direction increases, the calculated structural stresses stabilizes for all of the different meshes used.

Given this result, it is reasonable to assume that when only one element is used through the thickness then the calculated structural stress by the linearization procedure will also be dubious. It is likely that the main cause for this is that the mesh is not sufficiently fine to faithfully reproduce the stress distribution around the hot spot area.

Effect of averaging the nodal stresses at the weld toe

Figure A.2 shows a comparison of the structural stresses calculated with and without stress averaging at the weld toe. The differences that one can observe are significant. In the case of stress averaging the stresses obtained from the weld meshed with triangular shaped elements seem to be under-estimated when compared with stresses obtained using unaveraged results

On the other hand the stresses obtained from the weld meshed with quadrilateral shaped elements were less affected. In view of this result a further exercise was carried out in which the mesh size was gradually refined. The results tend to suggest that with mesh refinement the stresses obtained by the linearization procedure (with stress averaging) on the weld meshed with triangular shaped elements start to converge to those obtained from the weld meshed with quadrilateral elements. Interestingly the results of the latter have remained practically unaltered with mesh refinement showing that refinement was not necessary. It can therefore be confirmed that performing the stress linearization on the elements in front of the hot spot is clearly a better approach then using averaged stresses.

\section{Surface stress extrapolation}

Effect of the element shape

The use of different element shapes (quadrilateral and triangular) for the weld has some effect on the results obtained from surface extrapolation. This is mostly noticeable in the models where the number of elements along the surface (e_s) is either case A or case B (Fig. A.1). For cases of (e_s) being C, D or $\mathrm{E}$, the results are more consistent. This consistency was also noted during the mesh refinement exercise where the surface extrapolation results were practically unaltered in all of the cases.

\section{Element size and distortion}

As previously stated some of the combinations of number of elements through thickness and along the surface produce poor element shapes and consequently doubtful results (Tables A.1 and A.2). This is particularly evident in the results of the surface stress extrapolation when there is one element in the e $s_{1}$ region, i.e. between the hot spot and the first extrapolation point (Cases A and B in Table 1).

At e_t $=4$ and e_s $=$ A, B for the weld meshed with quadrilateral elements, Fig.A1, the values of the structural stress correlate well with the values obtained at finer meshes using surface extrapolation. The elements in these models are fairly distributed and therefore the good results may be attributed to this fact. However looking at another model $e_{-} t=$ 8 and e_s = A, Fig.A.1, this time for the weld meshed with triangular elements, the mesh is quite poor and yet the structural stress agrees with the values obtained at finer meshes using surface extrapolation. Therefore there seems to be a contradiction in the results.

Looking closely at Fig.A.1 a pattern in the value of structural stress obtained through surface extrapolation may be seen for the cases where $\mathrm{e}_{-} \mathrm{s}=\mathrm{A}$ and $\mathrm{B}$. It appears that as $\mathrm{e}_{-} \mathrm{t}$ is increasing (i.e. the mesh is refined in the thickness direction) the value of the structural stress is tending to decrease. This suggests that none of the results obtained from surface extrapolation at e_s $=\mathrm{A}$ and $\mathrm{B}$ are correct.

On the other hand, for e $\mathrm{t} \geq 4$ and for e $\mathrm{s}=\mathrm{C}, \mathrm{D}$ and $\mathrm{E}$, the results show a more valid trend. As the number of elements is increased in the thickness direction, for e $\mathrm{s}=\mathrm{C}, \mathrm{D}$ or $\mathrm{E}$, the extrapolation stress results remain relatively steady at a fixed value. Therefore it is reasonable to say that these results are acceptable because of their consistency. Furthermore, in all cases the meshes seem quite adequate (Tables A.1 and A.2) and so support the results obtained.

It can be observed that there is a tendency that the use of one element between the weld toe and the first extrapolation point together with more than one element in the thickness direction will under-predict the calculated stresses. As the number of elements in the thickness direction is increased the under prediction seems to increase. The extent to which the use of more than one element in the thickness direction together with only one element in the first extrapolation point negatively affects the calculated stresses gives reasons for concern. In the example considered in this paper the thickness of the plate was $10 \mathrm{~mm}$. Considering that the first extrapolation point is a 
distance of $0.4 t$ away from the weld toe, using two elements in the thickness direction would appear to be reasonable as this gives proportionately shaped elements $(4 \mathrm{~mm} \times 5 \mathrm{~mm})$.

From the results it seems fair to conclude that consistent extrapolation results can be obtained when there are at least two elements between the hot spot and the first extrapolation point and adequate elements size. With these conditions satisfied then the number of elements in the thickness direction would only be important to get good shaped elements with little distortion from the parent shape. This is in contrast to the IIW guidelines that recommend only one element in this region when solid elements are used [3].

\section{CONCLUSIONS}

In this paper a comparative study between three methods to calculate the structural stress was carried out. The three methods used are stress linearization through thickness, surface stress extrapolation and the nodal force (Dong's) method. For analyses purposes a T-shaped welded plate was considered.

In general it was noted that when using stress linearization through thickness and surface stress extrapolation the results can be mesh sensitive. On the other hand structural stresses calculated using the nodal force (Dong's) method show that the method is mesh insensitive for the meshes used for the models of the T-shaped welded plates under bending and the meshes considered in the paper.

When it comes to mesh density it can be said that the surface stress extrapolation method requires careful attention. In general, if more than one element is used in the thickness direction then more than one element should also be used along the surface, from the hot spot to the first extrapolation point and beyond.

On the other hand for linearization through thickness the effect of the mesh densities was less pronounced. When the mesh was too coarse the resulting stresses were overestimated. For linearization through thickness it is important that the calculations are based on the un-averaged stresses of the element in front of the hot spot. The results indicate that using averaged stresses can make the method highly mesh dependent.

It was noted that consistent extrapolation results can be obtained when there are at least two elements between the hot spot and the first extrapolation point and adequate elements size. This is in contrast to the IIW guidelines that recommend only one element in this region when solid elements are used [3].

The surface extrapolation and the stress linearization method can become quite cumbersome to use when free meshing is used in three dimensional models. Given that nodal forces can be obtained directly in the postprocessor of finite element software then the nodal force method has an advantage over the other two methods in terms of ease of use.

\section{ACKNOWLEDGMENT}

Use of the ANSYS finite element software through an educational license is acknowledged.

\section{REFERENCES}

[1] ASME Boiler and Pressure Vessel Code, Section VIII Division 2 Part 5 - 2007 Edition

[2] EN 13445 Part 3: Design 2006 - Unfired Pressure Vessels [3] Niemi E., Fricke W., Maddox S.J. Fatigue analysis of welded components, Designer's guide to the structural hot-spot approach, Woodhead Publishing Ltd, 2006

[4] Kroenke W. C. Classification of finite element stresses according to ASME Section III stress categories, Proc $94^{\text {th }}$ ASME Winter Annual Meeting, 1973.

[5] Kroenke W. C. et al, Interpretation of finite element stresses according to ASME III, ASME Tech. Paper 75-PVP-63, 1975.

[6] Gordon J. L. Outcur: An automated evaluation of two dimensional finite element stresses according to ASME, ASME Paper 76-WA/PVP-16, 1976.

[7] Design by Analysis Manual, published by the European Commission, Directorate General Joint Research Centre, Petten, The Netherlands, 1999.

[8] Poutiainen I, Tanskanen P, Marquis G., Finite element methods for structural hot spot stress determination - a comparison of procedures, Int. J Fatigue 2004; 26: 1147-1157

[9] Marshall P.W. Design of welded tubular connections. Basis and use of AWS code provisions, Developments in civil engineering, 37, Elsevier, 1992, pp.412.

[10] Niemi E. Stress determination for fatigue analysis of welded components, Abington Publishing, 1995, pp.1-6.

[11] Fricke W, Kahl A, Comparison of different structural stress approaches for fatigue assessment of welded ship structures, Marine structures 2005; 18, 473-488

[12] Doerk O, Fricke W, Weissenborn C, Comparison of different calculation methods for structural stresses at welded joints. Int. J Fatigue 2003; 25:359-369

[13] Dong P, A structural stress definition and numerical implementation for fatigue analysis of welded joints, Int. J Fatigue 2001; 23: 865-876

[14] ANSYS, Canonsburg, Pennsylvania, U.S.A 


\section{ANNEX A}

Table A.1 - T-shaped welded plates having the weld meshed with quadrilateral elements

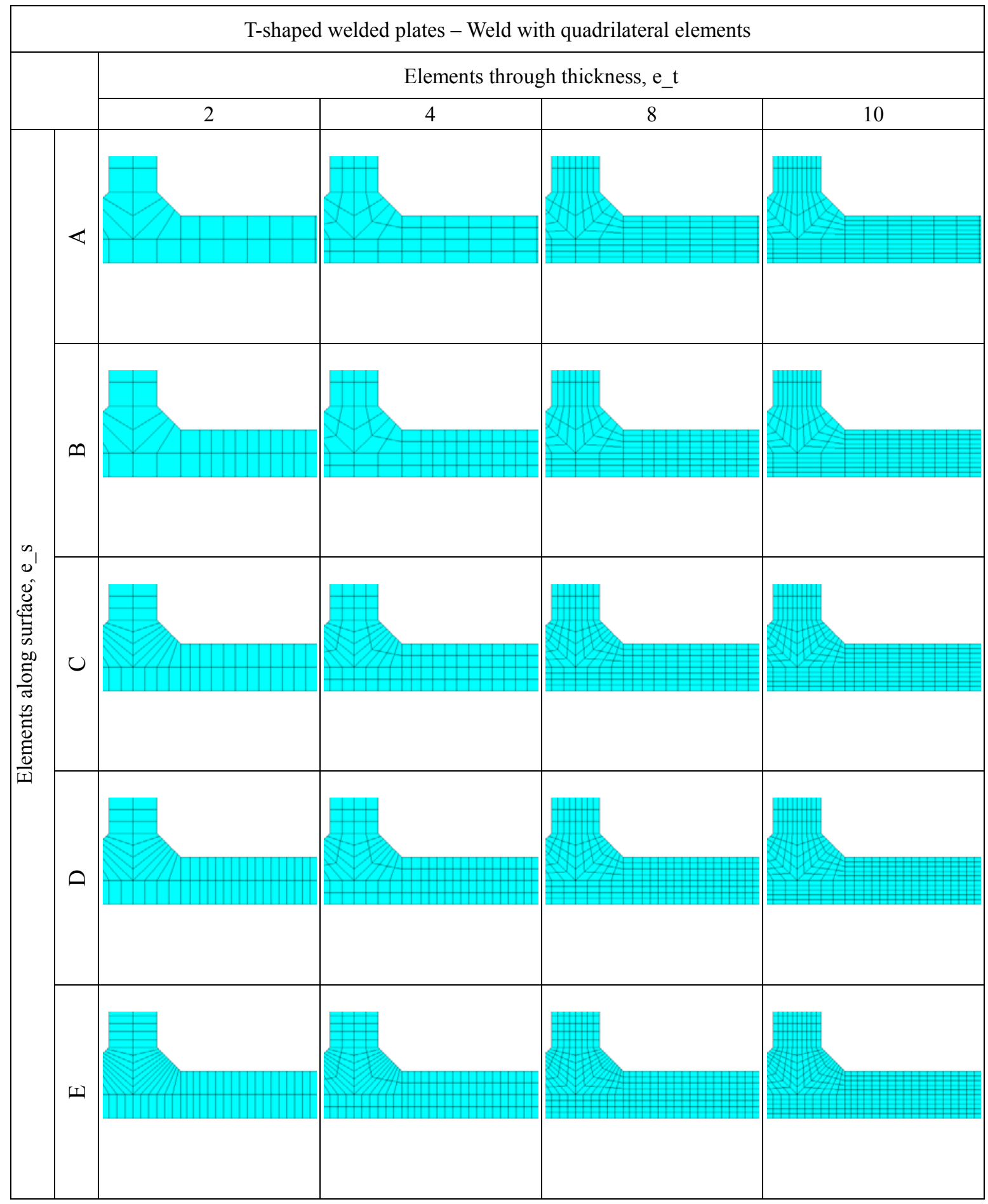


Table A.2 - T-shaped welded plates having the weld meshed with triangular elements

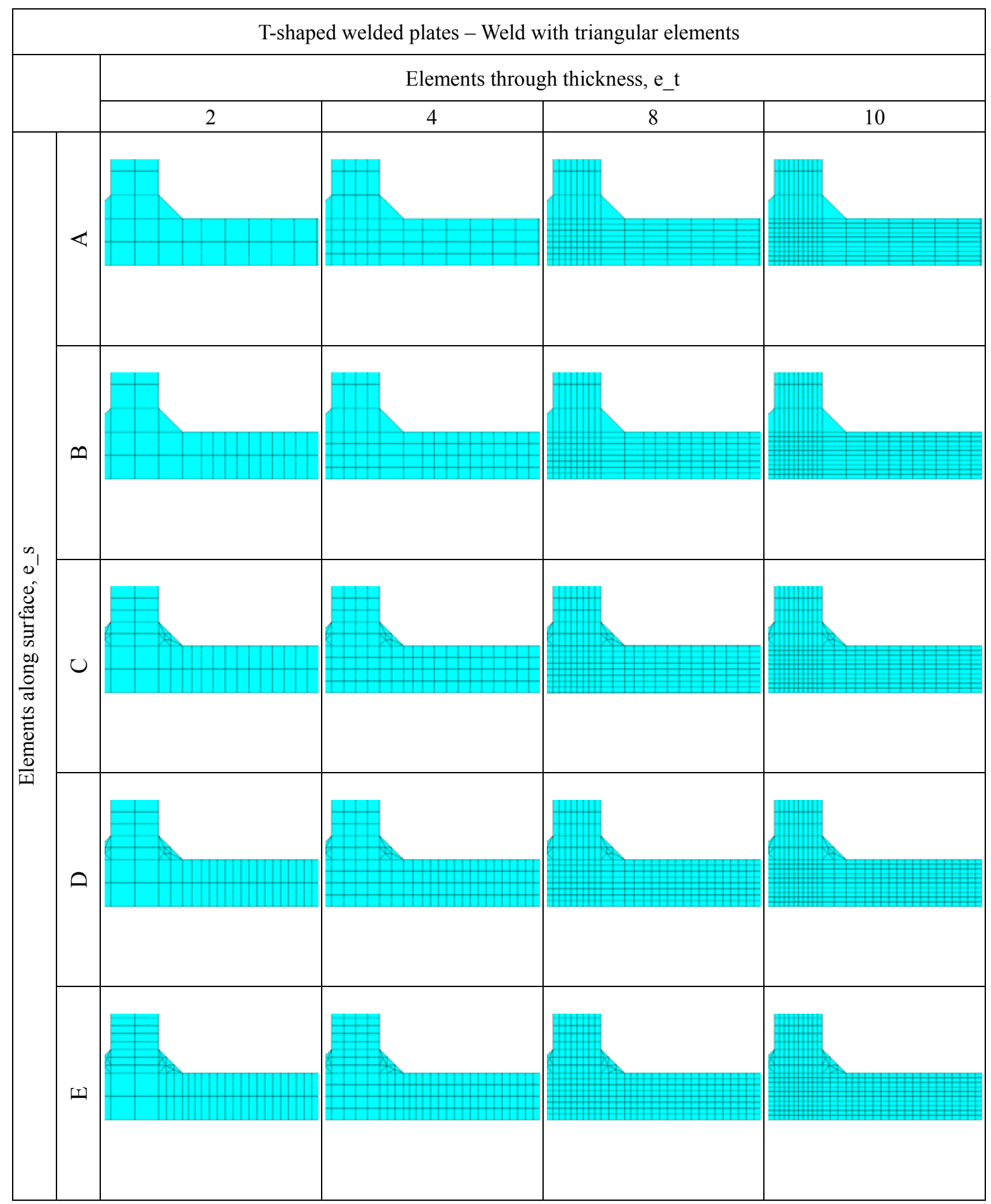




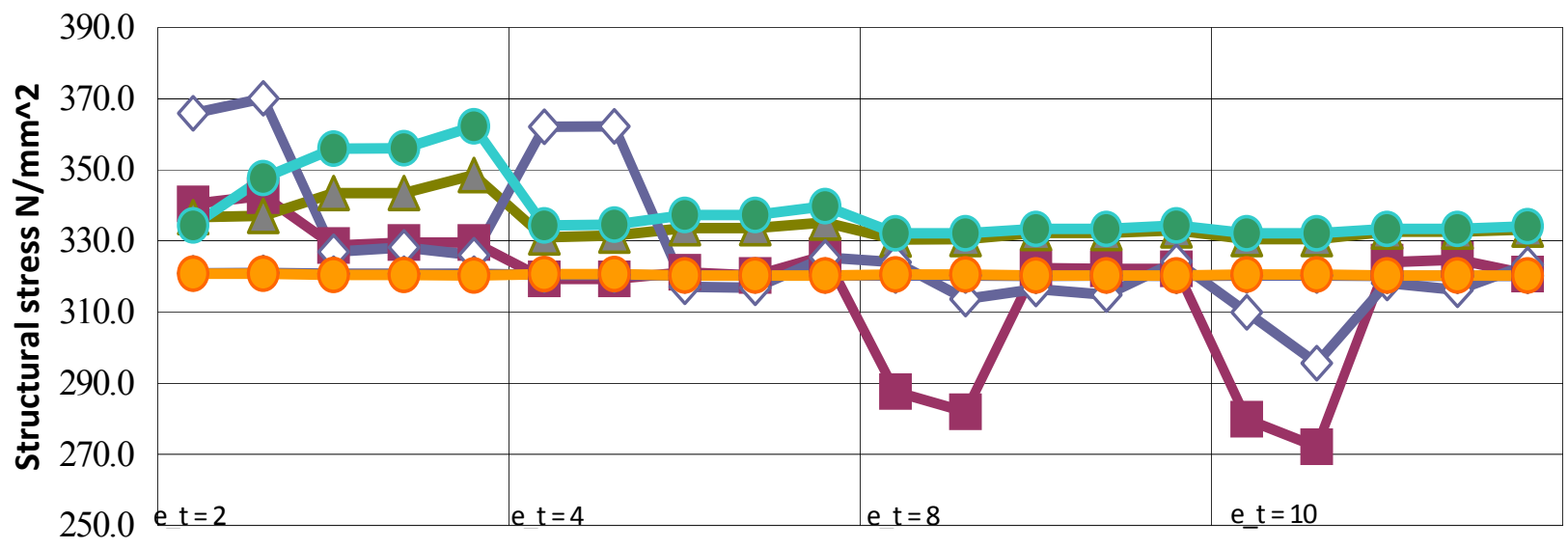
A B
C D
A B C
D
A $\quad$ B $\quad$ C D
D $\quad$ E $A$ B
D E

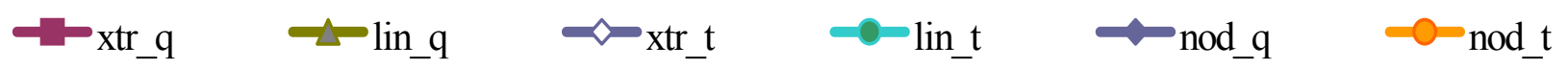

Figure A.1 - T-shaped welded plates - Structural stresses

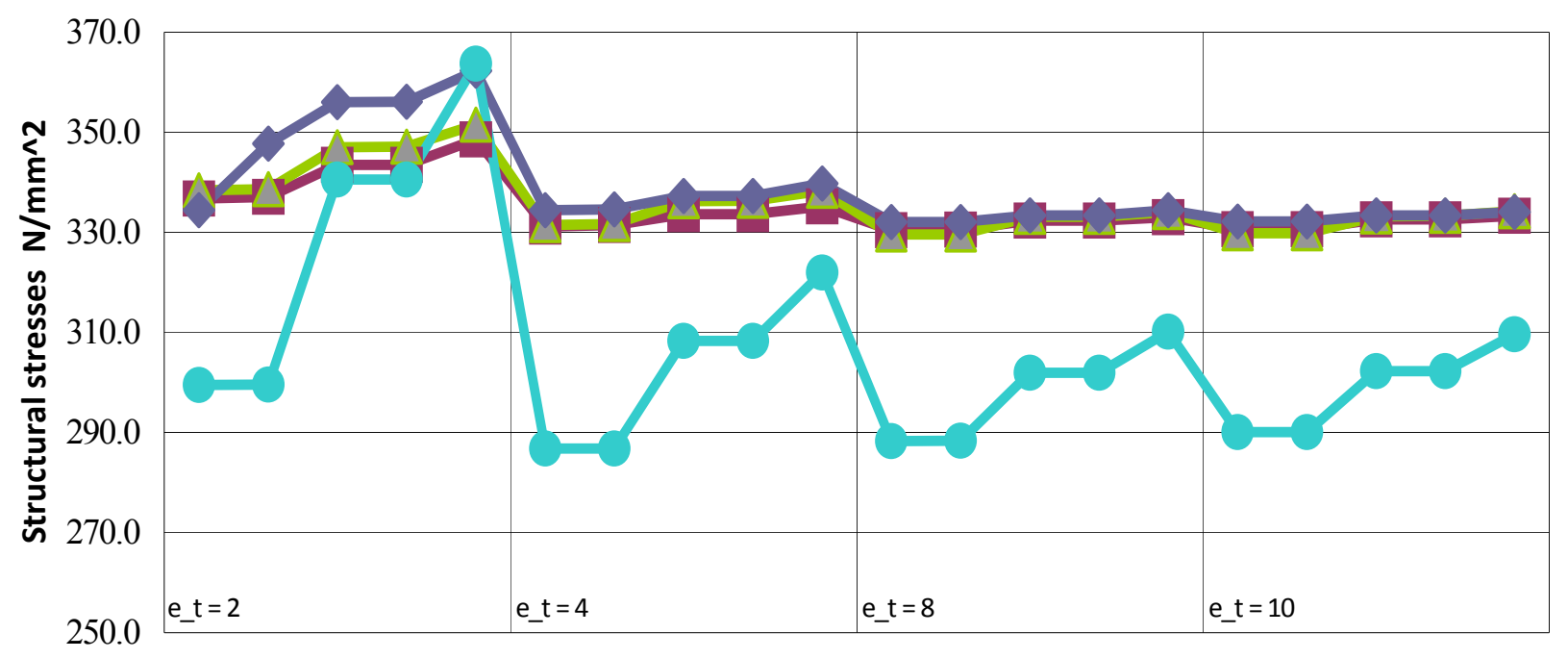

$\begin{array}{lllllllllllllllllllll}\mathrm{A} & \mathrm{B} & \mathrm{C} & \mathrm{D} & \mathrm{E} & \mathrm{A} & \mathrm{B} & \mathrm{C} & \mathrm{D} & \mathrm{E} & \mathrm{A} & \mathrm{B} & \mathrm{C} & \mathrm{D} & \mathrm{E} & \mathrm{A} & \mathrm{B} & \mathrm{C} & \mathrm{D} & \mathrm{E}\end{array}$ $\rightarrow$ lin_q noavg $\rightarrow$ lin_q avg lin_t_noavg -2 lin_avg

Figure A.2 - T-shaped welded plates - Structural stresses (averaged and unaveraged)- linearization through thickness 